\title{
Formation, evolution and multiplicity of brown dwarfs and giant exoplanets
}

José A. Caballero

\begin{abstract}
This proceeding summarises the talk of the awardee of the Spanish Astronomical Society award to the the best Spanish thesis in Astronomy and Astrophysics in the two-year period 2006-2007. The thesis required a tremendous observational effort and covered many different topics related to brown dwarfs and exoplanets, such as the study of the mass function in the substellar domain of the young $\sigma$ Orionis cluster down to a few Jupiter masses, the relation between the cluster stellar and substellar populations, the accretion discs in cluster brown dwarfs, the frequency of very low-mass companions to nearby young stars at intermediate and wide separations, or the detectability of Earth-like planets in habitable zones around ultracool (L- and T-type) dwarfs in the solar neighbourhood.
\end{abstract}

El que ama arde y el que arde vuela a la velocidad de la luz Lagartija Nick (Val del Omar)

\section{1 "De fuscis pusillis astris et giganteis exoplanetis" (Part I)}

The recipient of the Spanish Astronomical Society (Sociedad Española de Astronomia) award to the best Spanish thesis in Astronomy and Astrophysics in the two-year period 2006-2007 was the thesis "Formation, evolution and multiplicity of brown dwarfs and giant exoplanets" ("Formación, evolución y multiplicidad de enanas marrones y exoplanetas gigantes"), by the author of this proceeding. It was supervised by R. Rebolo and V. J. S. Béjar and defended at the Universidad de La Laguna/Instituto de Astrofísica de Canarias in March 2006.

José A. Caballero

Departamento de Astrofísica y Ciencias de la Atmósfera, Facultad de Física, Universidad Complutense de Madrid, E-28040 Madrid, Spain, e-mail:caballeroeastrax.fis.ucm.es 
My thesis was an ambitious initiative to search for the answers to some key questions in Astrophysics: How and where do the substellar objects form? What are their properties? How are they related to stars? Such answers should be obtained through observations at $1-10 \mathrm{~m}$-class telescopes, especially in the red optical and the nearinfrared. Just to illustrate the amount and variety of data eventually collected or the difficulty in summarising the thesis in a short talk or in this proceeding, during my PhD, I observed during 192 telescope nights with 18 different instruments in 11 different telescopes, not counting data aquired by other observers or with space missions (e.g. Hubble, Spitzer, XMM-Newton). I splitted the 459 pages of the thesis into five parts, 11 chapters and three appendices, which can be downloaded from a public ftp sitt 1 . Most of the chapters have been the basis of many refereed publications in main international journals. The used language was Spanish.

\subsection{Brown dwarfs and objects beyond the deuterium-burning mass limit (Chapter 1)}

This was the necessary introductory chapter of the thesis. It dealt with the following subjects:

- Physical properties of substellar objects: basic definitions, hydrogen and deuterium-burning mass limits, lithium test; time evolution of physical parameters (luminosity, temperature, absolute magnitudes, colours); ultracool atmospheres, new spectral types L and T, meteorology.

- An historical view of the searches of substellar objects (with an interesting discussion on which was the first brown dwarf: Teide 1 -Rebolo et al. 1995, 1996-, GJ 229 B -Nakajima et al. 1995-, PPL 15 -Stauffer et al. 1994; Basri et al. 1996, HD 114762 b -Latham et al. 1989-, GD 165B -Becklin \& Zuckerman 1988; Kirkpatrick et al. 1999- or LP 944-20 -Luyten \& Kowal 1975; Tinney 1998-);

- Theoretical scenarios of formation of substellar objects and planetary systems.

- Young star clusters, photometric searches and the substellar initial mass function.

- Ultracool companions to stars, multiplicity of L and T dwarfs, circumsubestellar discs (with compilations of late-type companions and very low-mass binaries).

The chapter ended with the main aims of the thesis, which were studying the mass function in the substellar domain of the $\sigma$ Orionis cluster $(\tau \sim 3 \mathrm{Ma}$ ) down to a few $M_{\text {Jup }}$, the relation between the cluster stellar and substellar populations, the accretion discs in young cluster brown dwarfs, the frequency of very low-mass companions to nearby young stars $(\tau \sim 100 \mathrm{Ma})$ at intermediate and wide separations, and the detectability of Earth-like planets in habitable zones around ultracool ( $\mathrm{L}$ and $\mathrm{T}$ ) dwarfs in the solar neighbourhood.

${ }^{1}$ ftp://astrax.fis.ucm.es/pub/users/caballero/PhD. 


\section{The substellar population in $\sigma$ Orionis and its relation with the stellar population (Part II)}

\subsection{The $\sigma$ Orionis cluster (Chapter 2)}

The fourth brightest star in the Orion Belt, about 2 mag fainter than the three main stars, is $\sigma$ Ori. The star, which is actually the hierarchical multiple Trapezium-like stellar system that illuminates the famous Horsehead Nebula, has taken a great importance in the last decade. Its significance lies in the very early spectral type of the hottest component $(\sigma$ Ori A, O9.5V) and in the homonymous star cluster that surrounds the system (Garrison 1967). The $\sigma$ Orionis star cluster, re-discovered due to its large number of X-ray emitters (Wolk 1996), contains one of the best known brown dwarf and planetary-mass object populations (Béjar et al. 1999, 2001; Zapatero Osorio et al. 2000, 2007; González-García et al. 2006), and is an excellent laboratory to study the evolution of X-ray emission, discs and angular momenta (Reipurth et al. 1998; Scholz \& Eislöffel 2004; Oliveira et al. 2006; Franciosini et al. 2006; Hernández et al. 2007; Skinner et al. 2008). Canonical, minimum and maximum values of main parameters of the cluster and some key references are provided in Table 1. In this chapter, I also described the work that the Canarias group had carried out in $\sigma$ Orionis, with an emphasis on the discovery and characterisation of S Ori 70, a mid-T-type object towards the cluster (Zapatero Osorio et al. 2002b, 2008; Martín \& Zapatero Osorio 2003; Burgasser et al. 2004; Scholz \& Jayawardhana 2008). Finally, I presented a compilation of cluster members with spectroscopic confirmation that was the basis of two published catalogues of stars and brown dwarfs in the $\sigma$ Orionis cluster (Caballero 2007a, 2008c).

Table 1 Main parameters of the $\sigma$ Orionis open cluster.

\begin{tabular}{lcccl}
\hline Parameter & $\begin{array}{c}\text { Canonical } \\
\text { value }\end{array}$ & $\begin{array}{c}\text { Min.:max. } \\
\text { values }\end{array}$ & Unit & Key references \\
\hline Age, $\tau$ & 3 & $1: 8$ & Ma & Zapatero Osorio et al. 2002a; Sacco et al. 2006 \\
Distance, $d$ & 385 & $330: 470$ & pc & Mayne \& Naylor 2008; Caballero 2008b \\
$E(B-V)$ & 0.07 & $0.00: 0.10$ & mag & Béjar et al. 2004b; Sherry et al. 2008 \\
{$[\mathrm{Fe} / \mathrm{H}]$} & $-0.02 \pm 0.13$ & $-0.15:+0.13$ & & González-Hernández et al. 2008 \\
Size, $r_{\max }$ & 30 & $20: 40$ & arcmin & Béjar et al. 2004a; Caballero 2008a \\
Total mass, $\Sigma M$ & $275^{a}$ & $150: 225$ & $M \odot$ & Sherry et al. 2004; Caballero 2007a \\
Disc frequency $^{b}$ & $\sim 33$ & $5:>50$ & $\%$ & Caballero et al. 2007; Luhman et al. 2008 \\
\hline
\end{tabular}

${ }^{a}$ The value of $\Sigma M=275 M_{\odot}$ is from Caballero (in prep.).

${ }^{b}$ The disc frequency in $\sigma$ Orionis is mass-dependent and increases towards lower masses. 


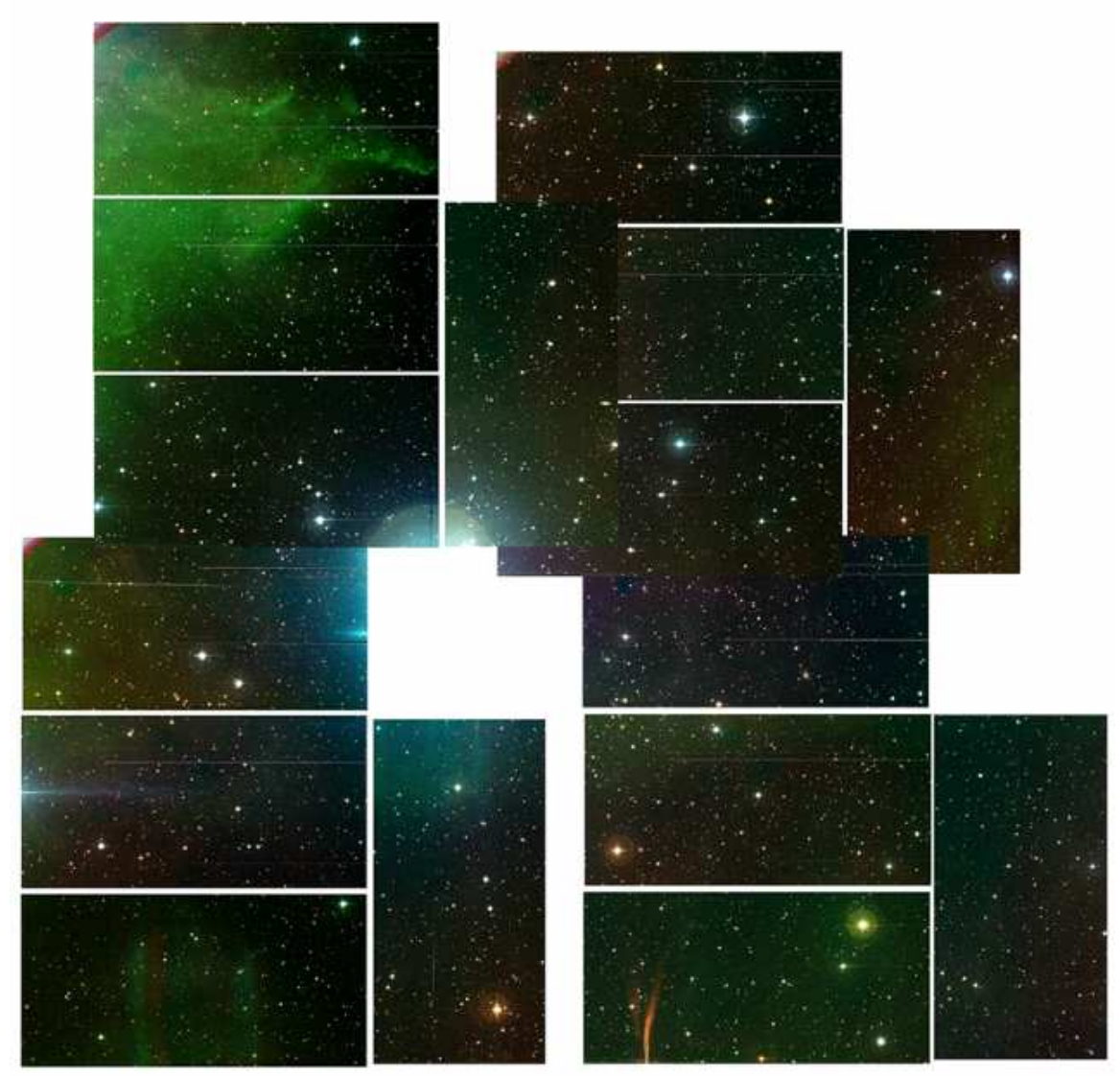

Fig. 1 False-colour mosaic of a region surrounding the $\sigma$ Orionis centre (the bright star $\sigma$ Ori falls in the central gap). The area corresponds to four pointings with the Wide Field Camera at the $2.5 \mathrm{~m}$ Isaac Newton Telescope. Colours red, green and blue are for passbands $I, R$ and $V$, respectively. Note the bright $R$-band (i.e. $\mathrm{H} \alpha$ ) emission to the northeast; the nebulosity is associated with the Horsehead Nebula.

\subsection{Multiobject spectroscopy in $\sigma$ Orionis: a bridge between the stellar and substellar populations (Chapter 3)}

We used the Wide Field Fibre Optical Spectrograph instrument and the robot positioner AutoFib2 (WYFFOS+AF2) at the $4.2 \mathrm{~m}$ William Herschel Telescope to aquire about 200 intermediate-resolution $(\mathrm{R} \sim 8000)$ spectra of sources in the direction of $\sigma$ Orionis. We covered the wavelength range between 6400 and $6800 \AA$. We compiled a list of 80 cluster members with WYFFOS+AF2 spectroscopy, based on the presence of $\mathrm{Li}$ I $\lambda 6707.8 \AA$ in absorption and $\mathrm{H} \alpha \lambda 6562.8 \AA$ in emission (late and mid-type stars) or spectral type determination (early-type stars). About one half 
of the objects were spectroscopically studied there for the first time. Using available data on the members, we investigated:

- the variation of the strength of the Li I line with spectral type (from $0.05 \AA$ in late F stars to $0.70 \AA$ in intermediate $\mathrm{M}$ stars), time and signal-to-noise ratio;

- the frequency of accretors according to the White \& Basri (2003) criterion $\left(46_{-13}^{+16} \%\right.$ of $\mathrm{K}$ and $\mathrm{M}$ stars - there might be a bias in the input sample towards $\mathrm{H} \alpha$ emitters) and the presence of asymmetries in the profiles of the $\mathrm{H} \alpha$ line;

- the existence of forbidden lines in emission ([N II] $\lambda \lambda 6548.0,6583.5 \AA$, [S II] $\lambda \lambda 6716.4,6730.8 \AA)$;

- the widening of photospheric lines (of up to $100 \mathrm{~km} \mathrm{~s}^{-1}$ ) due to fast rotation;

- the relationship between the $L^{\prime}$ - and $K_{\mathrm{s}}$-band flux excesses and the spectroscopic features associated with accretion from protoplanetary discs;

- the average of the radial velocity of the cluster members $\left(+30.2 \mathrm{~km} \mathrm{~s}^{-1}\right)$ and the existence of radial velocity outliers (probably due to unresolved close companions and contaminants of overlapping young stellar populations in the Orion Belt);

- and the frequency of X-ray emitters catalogued by ROSAT and ASCA space observatories as a function of spectral type (the bulk of the $\mathrm{K}$ stars are X-ray emitters).

Our WYFFOS+AF2 data were used in the analysis of chemical abundances of late-type pre-main sequence stars in $\sigma$ Orionis by González-Hernández et al. (2008), where we first determined the mean photospheric metallicity of the cluster. Besides, we presented a new Herbig-Haro object candidate (a few arcseconds to the southwest of the classical T Tauri star Mayrit 609206/2 and preliminary results on topics that have been developed afterwards, such as radial distribution (Caballero 2008a) and wide binarity (Caballero 2008d).

\subsection{A new mini-search in the centre of $\sigma$ Orionis (Chapter 4)}

Because of the intense brightness of the OB-type multiple star system $\sigma$ Ori, the low-mass stellar and substellar populations close to the centre of the very young $\sigma$ Orionis cluster was poorly know. I presented an $I J H K_{\mathrm{S}}$ survey in the cluster centre, able to detect from the massive early-type stars down to cluster members below the deuterium burning mass limit. The near-infrared and optical data were complemented with X-ray imaging with the XMM-Newton and Chandra space missions. Ten objects were found for the first time to display high-energy emission. Previously known stars with clear spectroscopic youth indicators and/or X-ray emission

2 Alternative names to Mayrit objects listed in this work, in order of appearance Mayrit 609206: V505 Ori; Mayrit 11238: $\sigma$ Ori C; Mayrit 13084: $\sigma$ Ori D; Mayrit 530005: S Ori J053847.5-022711; Mayrit 528005 AB: [W96] 4771-899; Mayrit 3020 AB: $\sigma$ Ori IRS1; Mayrit 306125 AB: HD 37525; Mayrit 208324: HD 29427; Mayrit 1359077: HD 37686; Mayrit 495216: S Ori J053825.4-024241. 
defined a clear sequence in the $I$ vs. $I-K_{\mathrm{S}}$ diagram. I found six new candidate cluster members that followed this sequence. One of them, in the magnitude interval of the brown dwarfs in the cluster, displayed X-ray emission and a very red $J-K_{\mathrm{S}}$ colour, indicative of a dis 3 . Other three low-mass stars have excesses in the $K_{\mathrm{s}}$ band as well. The frequency of X-ray emitters in the area is $80 \pm 20 \%$. The spatial density of stars is very high, of up to $1.6 \pm 0.1 \mathrm{arcmin}^{-2}$. There was no indication of lower abundance of substellar objects in the cluster centre. Finally, I also reported two cluster stars with X-ray emission located at only 8000-11000 AU to $\sigma$ Ori AFB, two sources with peculiar colours and an object with X-ray emission and near-infrared magnitudes similar to those of previously-known substellar objects in the cluster. (A near-infrared/optical/X-ray survey in the centre of $\sigma$ Orionis - Caballero 2007b)

\subsection{Multiplicity in $\sigma$ Orionis: adaptive optics in the near infrared (Chapter 5)}

Substellar objects, when companions to stars, are found in direct image at distances larger than $\sim 40 \mathrm{AU}$ to the primaries (e.g. Nakajima et al. 1995, Rebolo et al. 1998). While many multiple stellar systems and isolated substellar objects are found in the $\sigma$ Orionis cluster, no brown dwarf or planetary-mass object at projected physical separations from stellar members at less than about $10000 \mathrm{AU}$ has been published yet (but see the brown dwarf-exoplanet system candidate in Caballero et al. 2006b). Through a pilot programme of near-infrared adaptive optic imaging with Naomi+Ingrid at the William Herschel Telescope, we investigated the coronae between 150 and $7000 \mathrm{AU}$ from six stellar cluster members. The observed stars covered a wide range of spectral types, from $09.5 \mathrm{~V}$ to K7.0. Apart from the adaptive optic images, we used other near-infrared, optical and X-ray data to derive the real astrophysical nature of the detected visual companions. A total of 22 visual companions to the primary targets were detected in this pencil-beam survey. Six sources showed blue optical-near-infrared colours for their magnitudes, and they did not match in any colour-magnitude diagram of the cluster. There is not enough information to derive the nature of other five sources (including a faint object 2 arcsec northeast of Mayrit 11238; see Bouy et al. 2008). Eleven objects remained as cluster member candidates according to their magnitudes and colours: $(i)$ three of them were previously known cluster members: Mayrit 11238, Mayrit 13084 (surrounding $\sigma$ Ori AFB) and Mayrit 530005 (close to Mayrit $528005 \mathrm{AB}$ ); (ii) one is the near-infrared counterpart of the mid-infrared and radio source Mayrit $3020 \mathrm{AB}$, a dust cloud next to $\sigma$ Ori AFB discovered by van Loon \& Oliveira (2003). The object was also detected in Chandra archive images taken with the HRC-I instrument (this result was advanced in Caballero 2005; see again Bouy et al. 2008); (iii) one of the Mayrit $306125 \mathrm{AB}$ companions seemed to be a pre-main sequence photometric

3 This object is actually an emission-line, Type 1 , obscured quasar at $\mathrm{z}=0.2363 \pm 0.0005$ (UCM0536-0239; Caballero et al. 2002b). 
candidate star catalogued by Wolk (1996); (iv) two bright objects were the previously unknown secondaries of the Mayrit 306125 AB and Mayrit 528005 AB close binary systems, at angular separations of $0.45 \pm 0.04$ and $0.40 \pm 0.08$ arcsec, respectively; and $(v)$ the four remaining objects were visual companions to $\sigma$ Ori AFB (1), Mayrit 208324 (2) and Mayrit 1359077 (1) at separations from 5.5 to 19.0 arcsec. A few of them display features of youth (e.g. discs). Even if their common spatial velocities are measured in the future, it is not known whether the systems will survive the gravitational field of the young cluster.

\subsection{The mass function down to the planetary domain: the "Anaga" survey (Chapter 6)}

We investigated the mass function in the substellar domain of the $\sigma$ Orionis open cluster down to a few Jupiter masses. We performed a deep $I J$-band search with Isaac at the $8.2 \mathrm{~m}$ Very Large Telescope UT1 and the Wide Field Camera at the Isaac Newton Telescope, covering an area of $790 \mathrm{arcmin}^{2}$ close to the cluster centre. This survey was complemented with an infrared follow-up in the $H K_{\mathrm{S}^{-}}$and 3.6-8.0 $\mu \mathrm{m}$-bands with IRAC at the Spitzer Space Telescope, CFHT-IR at the $3.6 \mathrm{~m}$ Canada-France-Hawai'i Telescope, Omega-2000 at the $3.5 \mathrm{~m}$ Calar Alto Teleskop and CAIN-II at the $1.5 \mathrm{~m}$ Telescopio Carlos Sánchez. Using colour-magnitude diagrams, we selected 49 candidate cluster members in the magnitude interval $16.1 \mathrm{mag}$ $<I<23.0$ mag. Accounting for flux excesses at $8.0 \mu \mathrm{m}$ and previously known spectral features of youth, we identified 30 objects as bona fide cluster members. Four were first identified from our optical/near-infrared data. Eleven had most probable masses below the deuterium burning limit, which we therefore classified as candidate planetary-mass objects. The slope of the substellar mass spec$\operatorname{trum}\left(\Delta N / \Delta M \approx a M^{-\alpha}\right)$ in the mass interval $0.11 M_{\odot}<M<0.006 M_{\odot}$ is $\alpha=$ $+0.6 \pm 0.2$. Any mass limit to formation via opacity-limited fragmentation must lie below $0.006 M_{\odot}$. The frequency of $\sigma$ Orionis brown dwarfs with circumsubstellar

discs is $47 \pm 9 \%$. The continuity in the mass function and in the frequency of discs suggests that very low-mass stars and substellar objects, even below the deuteriumburning mass limit, share the same formation mechanism. Besides, the technique used for calculating in detail the back- and foreground contamination by field dwarfs of very late spectral types (intermediate and late $\mathrm{M}, \mathrm{L}$, and $\mathrm{T}$ ) that we presented in this chapter was developed by Caballero et al. (2008a) with the adoption of the latest models from the literature. (The substellar mass function in $\sigma$ Orionis. II. Optical, near-infrared and IRAC/Spitzer photometry of young cluster brown dwarfs and planetary-mass objects - Caballero et al. 2007) 


\section{Activity and meteorology in ultracool objects: discs and atmospheres (Part III)}

\subsection{Photometric variability of young brown dwarfs in $\sigma$ Orionis (Chapter 7)}

We carried out multi-epoch, time-series differential $I$-band photometry of a large sample of objects in the south-east region of the $\sigma$ Orionis open cluster. A field of $\sim 1000 \mathrm{arcmin}^{2}$ was monitored with the Wide Field Camera at the Isaac Newton Telescope during four nights over a period of two years. Using this dataset, we studied the photometric variability of twenty-eight brown dwarf cluster candidates with masses ranging from the stellar-substellar boundary down to the planetary-mass domain. We found that about $50 \%$ of the sample showed photometric variability on timescales from less than one hour to several days and years. The amplitudes of the $I$-band light curves ranged from less than 0.01 up to $\sim 0.4$ mag. A correlation between the near-infrared excess in the $K_{\mathrm{S}}$ band, strong $\mathrm{H} \alpha$ emission and largeamplitude photometric variation was observed. We briefly discussed how these results may fit the different scenarios proposed to explain the variability of cool and ultracool dwarfs (i.e. magnetic spots, patchy obscuration by dust clouds, surrounding accretion discs and binarity). Additionally, we determined tentative rotational periods in the range 3 to $40 \mathrm{~h}$ for three objects with masses around $60 M_{\mathrm{Jup}}$, and the rotational velocity of $14 \pm 4 \mathrm{~km} \mathrm{~s}^{-1}$ for one of them. The shortest periods can be explained by pulsational instability excited by central deuterium burning during the initial phases of evolution of young brown dwarfs. (Photometric variability of young brown dwarfs in the $\sigma$ Orionis open cluster - Caballero et al. 2004)

\subsection{S Ori J053825.4-024241: a classical T Tauri-like object at the substellar boundary (Chapter 8)}

We presented a spectrophotometric analysis of Mayrit 495216 (S Ori J053825.4024241), a candidate member close to the substellar boundary of the $\sigma$ Orionis cluster. Our optical and near-infrared photometry and low-resolution spectroscopy indicated that Mayrit 495216 is a likely cluster member with a mass estimated from evo-

lutionary models at $0.06_{-0.02}^{+0.07} M_{\odot}$, which made the object a probable brown dwarf. The radial velocity of Mayrit 495216 was similar to the cluster systemic velocity. This target, which we classified as an M 6.0 \pm 1.0 low-gravity object, showed excess emission in the near-infrared and anomalously strong photometric variability for its type (from the blue to the $J$ band), suggesting the presence of a surrounding disc. The optical spectroscopic observations showed a continuum excess at short wavelengths and a persistent and resolved $\mathrm{H} \alpha$ emission (pseudo-equivalent width of about $-250 \AA$ ) in addition to the presence of other forbidden and permitted emission lines, which we interpret as indicating accretion from the disc and possibly 
mass loss. We concluded that despite the low mass of Mayrit 495216, this object exhibits some of the properties typical of active classical T Tauri stars. [Facilities: LRIS at the $10.0 \mathrm{~m}$ Keck I Telescope, ALFOSC at the $2.6 \mathrm{~m}$ Nordic Optical Telescope, CAIN-II at the Telescopio Carlos Sánchez, ESACCD at the $1.0 \mathrm{~m}$ European Space Agency Optical Ground Station, CCD\#1 at the $0.8 \mathrm{~m}$ Telescopio IAC80] (S Ori J053825.4-024241: a classical T Tauri-like object at the substellar boundary - Caballero et al. 2006a)

\section{Very low-mass companions to young stars and ultracool dwarfs in the solar nighbourhood (Part IV)}

\subsection{A search for very low-mass objects around nearby young stars (Chapter 9)}

There is a strong competition for searching for and characterising resolved brown dwarfs and exoplanets in orbit to neighbour stars, being the ultimate goal of many astronomers the imaging of exoearths in solar-like systems. Given the overluminosity of very low mass objects during their contraction phase, most high spatialresolution, photo(astro)metric searches have tended to explore nearby $(d<100 \mathrm{pc})$, young ( $\tau \sim 10-600 \mathrm{Ma}$ ) stars (e.g. Neuhäuser et al. 2003; McCarthy \& Zuckerman 2004; Metchev \& Hillenbrand 2004; Masciadri et al. 2005; Lowrance et al. 2005; Biller et al. 2007; Lafrenière et al. 2007; Carson et al. 2008). Following this idea, we imaged 51 stellar systems with features of youth (lithium, chromospheric activity, $\mathrm{X}$-ray emission, membership in moving group) with the NICMOS instrument and the coronograph at the Hubble Space Telescope and with near infrared adaptive optics systems attached at $4 \mathrm{~m}$-class telescopes: Alfa+Omega-Cass at the $3.5 \mathrm{~m}$ Calar Alto Teleskop, AdOpt@TNG+NICS at the $3.6 \mathrm{~m}$ Telescopio Nazionale Galileo and, especially, Naomi+Ingrid at the William Herschel Telescope. High resolution images were complemented with wide-field searches with CAIN-II at the Telescopio Carlos Sánchez and other near-infrared and optical instruments. Of the 51 investigated systems, 32 (44) are $100 \mathrm{Ma}$ (600 Ma) old or younger. The survey was designed to detect all brown dwarfs at projected physical separations $\Delta>50 \mathrm{AU}$ and all exoplanets with $M_{2}>0.008 M_{\odot}$ at $\Delta>100 \mathrm{AU}$. However, we did not detect any new substellar object. Complementing our results with those in the literature, the frequency of substellar objects with $M_{2}>0.008 M_{\odot}$ is less than $2 \%$ at any distance interval. Besides, we discovered three, possibly four, new stellar companions $\left(M_{2} \sim 0.35-0.80 M_{\odot}\right)$ and measured accurate astrometry $(\rho, \theta)$ of a dozen young, late-type, close binaries. From a personal point of view, the search was characterised by our "bad luck": AB Dor C (Close et al. 2005) was below the NICMOS coronographic mask; HN Peg B (Luhman et al. 2007) was out of the Naomi+Ingrid field of view and had no optical images to complement with our wide-field near-infrared 
ones; the individual exposure time for the binary HD 160934 AC (Hormuth et al. 2007) was too long and we could not resolve it, etc.

\subsection{Multiplicity of L dwarfs: binarity and habitable planets (Chapter 10)}

On the one hand, stars do have planets. The least massive exoplanets found to date, with a few Earth masses $\left(M_{\oplus}\right)$, orbit low-mass, M-type stars. On the other hand, the most massive moons in the Solar System, with up to $0.025 M_{\oplus}$, orbit giant planets. Brown dwarfs, with masses in between the least massive stars and the most massive giant planets, also have planets in wide (Chauvin et al. 2004) and close orbits (Joergens \& Müller 2007). Besides, the frequency of (protoplanetary) discs in brown dwarfs is comparable, or even larger, than in stars (see, for example, Caballero et al. 2007; Section 2.5. Therefore, it is natural to hypothesise the existence of terrestrial planets surrounding very low-mass stars and brown dwarfs with spectral types $\mathrm{L}$ and T. Because of their intrinsic dimmness, the habitable zones are very close to the Roche limit of the central objects. Such kind of systems can be detected with current technology. In this chapter, I showed preliminary results on photometric monitoring at medium-size telescopes to search for transits (Caballero \& Rebolo 2002; Blake et al. 2008), that resulted in a search for variability in brown dwarf atmospheres and for wide faint companions (Goldman 2003; Caballero et al. 2003; Goldman et al. 2008), and detailed a methodology for detecting exoearths in habitable zones around nearby L (and T) dwarfs with high-resolution near-infrared spectrographs (e.g. Nahual at the $10.4 \mathrm{~m}$ Gran Telescopio Canarias).

\section{Conclusions, appendices and bibliography (Part V)}

\subsection{Summary (Chapter 11)}

As a corollary of my thesis, the frequency of substellar companions is low, whether around nearby stars in the field or whether close to very young stars in the $\sigma$ Orionis cluster. However, isolated brown dwarfs and planetary-mass objects in clusters represent a significative fraction of the total number of objects (but not of the total mass). The similarity in spatial distribution and the continuity in the rising mass spectrum and in the frequency of discs suggest that very low-mass stars and substellar objects, even below the deuterium-burning mass limit, share the same formation mechanism. If they formed in protoplanetary discs by gravitational instabilities, a very efficient ejection mechanism would be necessary during the first few million years. Thus, the isolated planetary-mass objects that we find free-floating in clusters likely formed from turbulent fragmentation in the primigenious gas cloud. 
This thesis is a "full stop, new sentence" in the quotation Smaller, Fainter, Cooler (in humorous contraposition to Bigger, Stronger, Faster) of the brown dwarf and exoplanet searches.

Acknowledgements I thank R. Rebolo and V. J. S. Béjar for helpful comments an innumerable individuals and groups for their friendship and assistance during my PhD. Especial gratitude is for $u K i$ and M4M4. Most of the thesis research was conducted during my residence at the Instituto de Astrofísica de Canarias. Partial financial support was provided by a number of projects of the Spanish Ministerio Educación y Ciencia, Ministerio de Ciencia y Tecnología, Comunidad Autónoma de Madrid, Universidad Complutense de Madrid, Spanish Virtual Observatory, and European Social Fund.

\section{References}

1. G. Basri, G. W. Marcy, J. R. Graham, ApJ 458, 600 (1996).

2. E. E. Becklin, B. Zuckerman, Nature 336, 656 (1988).

3. V. J. S. Béjar, M. R. Zapatero Osorio, R. Rebolo, ApJ 521, 671 (1999).

4. V. J. S. Béjar, E. L. Martín, M. R. Zapatero Osorio et al., ApJ 556, 830 (2001).

5. V. J. S. Béjar, J. A. Caballero, R. Rebolo, M. R. Zapatero Osorio, D. Barrado y Navascués, Ap\&SS 292, 339 (2004a).

6. V. J. S. Béjar, M. R. Zapatero Osorio, R. Rebolo, AN 325, 705 (2004b).

7. B. A. Biller, L. M. Close, E. Masciadri et al., ApJS 173, 143 (2007).

8. C. H. Blake, J. S. Bloom, D. W. Latham, et al., PASP 120, 860 (2008).

9. H. Bouy, N. Huélamo, E. L. Martín et al., A\&A in press, arXiv:0808.3890(2008).

10. A. J. Burgasser, J. D. Kirkpatrick, M. R. McGovern et al., ApJ 604, 827 (2004).

11. J. A. Caballero, $A N$ 326, 1007 (2005).

12. J. A. Caballero, $A \& A$ 466, 917 (2007a).

13. J. A. Caballero, $A N$ 328, 917 (2007b).

14. J. A. Caballero, MNRAS 383, 375 (2008a).

15. J. A. Caballero, MNRAS 383, 750 (2008b).

16. J. A. Caballero, $A \& A$ 478, 667 (2008c).

17. J. A. Caballero, "Highlights of Spanish Astrophysics V", Proceedings of the VIII Scientific Meeting of the Spanish Astronomical Society. Santander, Spain, 7-11 July 2008. Eds. J. Gorgas, L. J. Goicoechea, J. I. González-Serrano, J. M. Diego. Poster \#103 (2008d).

18. J. A. Caballero, R. Rebolo, Proceedings of the First Eddington Workshop on Stellar Structure and Habitable Planet Finding. Córdoba, Spain, 11-15 June 2001. Eds. B. Battrick, F. Favata, I. W. Roxburgh, D. Galadí. ESA SP-485, Noordwijk: ESA Publications Division, ISBN $92-$ 9092-781-X, 261 (2002).

19. J. A. Caballero, V. J. S. Béjar, R. Rebolo, “Brown Dwarfs”, Proceedings of IAU Symposium \#211. University of Hawai'i, Honolulu, Hawai'i, 20-24 May 2002. Ed. E. L. Martín. San Francisco: Astronomical Society of the Pacific, 455 (2003).

20. J. A. Caballero, V. J. S. Béjar, R. Rebolo, M. R. Zapatero Osorio, A\&A 424, 857 (2004).

21. J. A. Caballero, E. L. Martín, M. R. Zapatero Osorio et al., A\&A 445, 143 (2006a).

22. J. A. Caballero, E. L. Martín, P. D. Dobbie, D. Barrado y Navascués, A\&A 460, 635 (2006b).

23. J. A. Caballero, V. J. S. Béjar, R. Rebolo et al., $A \& A$ 470, 903 (2007).

24. J. A. Caballero, A. J. Burgasser, R. Klement, $A \& A$ 488, 181 (2008a).

25. J. A. Caballero, L. Valdivielso, E. L. Martín et al., A\&A in press, DOI: 10.1051/00046361:200810616, arXiv:0809.0996 (2008b).

26. J. C. Carson, K. D. Hiner, G. G. Villar III et al., $A J$ in press, eprint arXiv:0810.1723 (2008).

27. G. Chauvin, A.-M. Lagrange, C. Dumas et al., A\&A, 425, L29 (2004). 
28. L. M. Close, R. Lenzen, J. C. Guirado et al., Nature, 433, 286 (2005).

29. E. Franciosini, R. Pallavicini, J. Sanz-Forcada, $A \& A$, 446, 501 (2006).

30. R. F. Garrison, PASP, 79, 433 (1967).

31. B. Goldman, The CLOUDS Collaboration, "Brown Dwarfs", Proceedings of IAU Symposium \#211. University of Hawai'i, Honolulu, Hawai'i, 20-24 May 2002. Ed. E. L. Martín. San Francisco: Astronomical Society of the Pacific, 461 (2003).

32. B. Goldman, M. C. Cushing, M. S. Marley et al., A\&A, 487, 277 (2008).

33. B. M. González-García, M. R. Zapatero Osorio, V. J. S. Béjar et al., A\&A, 460, 799 (2006).

34. J. I. González Hernández, J. A. Caballero, R. Rebolo et al., A\&A in press, DOI: 10.1051/00046361:200810398, arXiv:0809.0090 (2008).

35. J. Hernández, L. Hartmann, T. Megeath et al., ApJ 662, 1067 (2007).

36. F. Hormuth, W. Brandner, S. Hippler, M. Janson, T. Henning, A\&A 463, 707 (2007).

37. V. Joergens, A. Müller, ApJ 666, L113 (2007).

38. J. D. Kirkpatrick, F. Allard, T. Bida et al., ApJ 519, 834 (1999).

39. D. Lafrenière, R. Doyon, C. Marois et al., ApJ, 670, 1367 (2007).

40. D. W. Latham, R. P. Stefanik, T. Mazeh, M. Mayor, G. Burki, Nature, 339, 38 (1989).

41. J. Th. van Loon, J. M. Oliveira, A\&A, 405, L33 (2003).

42. P. J. Lowrance, E. E. Becklin, G. Schneider et al., AJ, 130, 1845 (2005).

43. K. L. Luhman, B. M. Patten, M. Marengo et al., ApJ, 654, 570 (2007).

44. K. L. Luhman, J. Hernández, J. J. Downes, L. Hartmann, C. Briceño, ApJ in press, arXiv:0808.0471 (2008).

45. W. J. Luyten, C. T. Kowal, Proper motion survey with forty-eight inch Schmidt Telescope. XLIII. One hundred and six faint stars with large proper motions. University of Minnesota, Minneapolis (1975).

46. C. McCarthy, B. Zuckerman, AJ 127, 2871 (2004).

47. E. L. Martín, M. R. Zapatero Osorio, ApJ 593, L113 (2003).

48. E. Masciadri, R. Mundt, T. Henning, C. Álvarez, D. Barrado y Navascués, ApJ 625, 1004 (2005).

49. N. J. Mayne, T. Naylor, MNRAS 386, 261 (2008).

50. S. A. Metchev, L. A. Hillenbrand, ApJ 617, 1330 (2005).

51. T. Nakajima, B. R. Oppenheimer, S. R. Kulkarni et al., Nature 378, 463 (1995).

52. R. Neuhäuser, E. W. Guenther, J. Alves et al., AN 324, 535 (2003).

53. J. M. Oliveira, R. D. Jeffries, J. Th. van Loon, M. T. Rushton, MNRAS 369, 272 (2006).

54. R. Rebolo, M. R. Zapatero Osorio, E. L. Martín, Nature 377, 129 (1995).

55. R. Rebolo, E. L. Martín, G. Basri et al., ApJ 469, L53 (1996).

56. R. Rebolo, M. R. Zapatero Osorio, S. Madruga et al., Science 282, 1309 (1998).

57. Bo Reipurth, J. Bally, R. A. Fesen, D. Devine, Nature 396, 343 (1998).

58. G. G. Sacco, E. Franciosini, S. Randich, R. Pallavicini, A\&A 488, 167 (2008).

59. A. Scholz, J. Eislöffel, $A \& A$ 419, 249 (2004).

60. A. Scholz, R. Jayawardhana, ApJ 672, L49 (2008).

61. W. H. Sherry, F. M. Walter, S. J. Wolk, AJ 128, 2316 (2004).

62. W. H. Sherry, F. M. Walter, S. J. Wolk, N. R. Adams, AJ 135, 1616 (2008).

63. S. L. Skinner, K. R. Sokal, D. H. Cohen et al., ApJ 683, 796 (2008).

64. J. R. Stauffer, D. Hamilton, R. G. Probst, $A J$ 108, 155 (1994).

65. C. G. Tinney, MNRAS 296, L42 (1998).

66. R J.White, G. Basri, ApJ 582, 1109 (2003).

67. S. J. Wolk, PhD thesis, State University New York at Stony Brook, NY, USA (1996).

68. M. R. Zapatero Osorio, V. J. S. Béjar, E. L. Martín et al., Science 290, 103 (2000).

69. M. R. Zapatero Osorio, V. J. S. Béjar, Ya. Pavlenko et al., A\&A 384, 937 (2002a).

70. M. R. Zapatero Osorio, V. J. S. Béjar, E. L. Martín et al., ApJ 578, 536 (2002b).

71. M. R. Zapatero Osorio, J. A. Caballero, V. J. S. Béjar et al., A\&A 472, L9 (2007).

72. M. R. Zapatero Osorio, V. J. S. Béjar, G. Bihain et al., A\&A 477, 895 (2008). 